\title{
AN OPTIMAL METHOD FOR THE COMPUTATION OF AN APPROXIMATE SOLUTION OF A CLASS OF DIFFERENTIAL EQUATIONS WITH APPLICATION IN ECONOMY
}

\author{
BOTA, C[onstantin]; CARUNTU, B[ogdan] \& BOTA, C[iprian]
}

\begin{abstract}
We propose a new optimal collocation-type method used to compute an approximate analytical polynomial solution for a class of nonlinear differential equations with boundary or initial conditions, and we employ this method for the case of a numerical example with applications in economy. This method modifies the collocation method by replacing the differentialtype conditions with integral ones and by including an optimization step.

Key words: nonlinear differential equations, boundary value problem, analytical approximate polynomial solution
\end{abstract}

\section{INTRODUCTION}

Many problems in economics can be modeled using nonlinear differential equations with boundary or initial conditions of the type :

$$
\begin{gathered}
x^{(2)}(t)=F\left(x^{(1)}(t), x(t), t\right), a \leq t \leq b \\
g_{i}\left(x^{(1)}(a), x^{(1)}(b), x(a), x(b), a, b\right)=0, \quad i=1,2
\end{gathered}
$$

where $F, g_{i}$ are continuous differentiable real functions.

Such problems were studied for example in (Chen et al., 1989; Sahmsul Alam et al. 2006). Usually, for problems of the type $(1,2)$, it is difficult, and sometimes impossible to find an exact solution, hence a numerical solution or an approximate analytical solution must be found, by using various methods such as : methods based on Taylor polynomials and Chebyshev polynomials (Chena et al., 2005), homotopy methods (He, 2003), collocation-type methods (Gotovac et al., 2009).

In this paper we will use a new method to solve a problem of the type $(1,2)$ and apply this method to find approximate solutions for a problem with applications in economy.

We consider the operator $D(x)=x^{(2)}(t)-F\left(x^{(1)}(t), x(t), t\right)$.

$\mathrm{We}$ are interested in finding approximate polynomial solutions $x_{a p p}$ on the $[\mathrm{a}, \mathrm{b}]$ interval, solutions which satisfy the following condition :

$$
|R(t)|<\varepsilon
$$

where

$$
R(t)=D\left(x_{a p p}(t)\right), t \in[a, b],
$$

represents the error obtained by replacing in (1) the exact solution $x$ with the polynomial approximation $x_{a p p}$.

We call $\varepsilon$ - approximate polynomial solution of the problem $(1,2)$ an approximate polynomial solution $x_{a p p}$ satisfying the relation (3).

The collocation method determines the approximating polynomial by imposing that it satisfies, beside the initial conditions (such as (2)), the condition that the derivatives of this polynomial are equal to the derivatives of the exact solution of the problem $(1,2)$ in certain points from the $[a, b]$ interval.
This condition, which is imposed in order to determine the coefficients of the polynomial, is rather restrictive when we wish to determine an approximate polynomial solution of the type presented above, which satisfies (3). In our method we replace this condition with an integral condition which allows for the calculation of the approximating polynomial with the desired accuracy (in the sense of (3)).

\section{THE OPTIMAL COLLOCATION METHOD}

We consider the problem $(1,2)$ where the real functions $F, g_{i}$ are continuously differentiable.

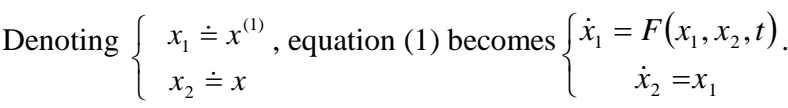

As a more concise notation, we use

$$
\dot{x}_{j}=f_{j}\left(x_{1}, x_{2}, t\right), j=1,2,
$$

where $f_{1}=F\left(x_{1}, x_{2}, t\right), f_{2}=x_{1}$.

We consider a discretization of the time interval $a=t_{1}<t_{2}<\ldots<t_{p}=b$, where $t_{j+1}-t_{j}=h, j=1, \ldots, p-1$.

First we will find an approximation $x_{a p p}$ for the solution of eq. (1) with the conditions (2), approximation which makes use of continuous functions, piecewise defined as polynomials between $x\left(t_{j}\right)$ and $x\left(t_{j+1}\right)$. We choose :

$x_{\text {app }}(t)=\sum_{k=0}^{m} c_{k}^{j}\left(\frac{t-t_{j}}{h}\right)^{k}, t \in\left[t_{j}, t_{j+1}\right], \quad j=1, \ldots, p-1, m \geq 2$

By imposing for $x_{a p p}$ the condition that it satisfies the differential equation (1) in the endpoints of the intervals $\left[t_{j}, t_{j+1}\right], j=1, \ldots, p-1$, and denoting the approximate values of the derivatives in $t_{j}, \frac{t_{j}+t_{j+1}}{2}, t_{j+1}$ by $d\left(t_{j}\right), d\left(\frac{t_{j}+t_{j+1}}{2}\right), d\left(t_{j+1}\right)$ respectively, we calculate the corresponding values of the coefficients $c_{k}^{j}$.

In the case $\boldsymbol{m}=\mathbf{5}$ we find the coefficients :

$$
\begin{gathered}
c_{0}=x\left(t_{j}\right), \quad c_{1}=h d\left(t_{j}\right), \\
c_{2}=\frac{1}{4}\left(-23 x\left(t_{j}\right)-12 h d\left(t_{j}\right)+7 x\left(t_{j+1}\right)-2 h d\left(t_{j+1}\right)\right. \\
+16 x\left(\frac{t_{j}+t_{j+1}}{2}\right)-16 d\left(\frac{t_{j}+t_{j+1}}{2}\right) \\
c_{3}=\frac{1}{4}\left(33 x\left(t_{j}\right)+13 h d\left(t_{j}\right)-17 x\left(t_{j+1}\right)+5 h d\left(t_{j+1}\right)\right. \\
-16 x\left(\frac{t_{j}+t_{j+1}}{2}\right)+32 d\left(\frac{t_{j}+t_{j+1}}{2}\right)
\end{gathered}
$$




$$
\begin{gathered}
c_{4}=\frac{1}{4}\left(-17 x\left(t_{j}\right)-6 h d\left(t_{j}\right)+13 x\left(t_{j+1}\right)-4 h d\left(t_{j+1}\right)\right. \\
+4 x\left(\frac{t_{j}+t_{j+1}}{2}\right)-20 d\left(\frac{t_{j}+t_{j+1}}{2}\right) \\
c_{5}=\frac{1}{4}\left(3 x\left(t_{j}\right)+h d\left(t_{j}\right)-3 x\left(t_{j+1}\right)+h d\left(t_{j+1}\right)+4 d\left(\frac{t_{j}+t_{j+1}}{2}\right)\right. \\
\text { where } h=\frac{t_{j+1}-t_{j}}{2} .
\end{gathered}
$$

By replacing the values of these coefficients in (5) we obtain the approximate polynomial solutions $x_{a p p}$ as a function of the unknowns

$$
\begin{gathered}
x\left(t_{j}\right), \quad x\left(\frac{t_{j}+t_{j+1}}{2}\right), \quad x\left(t_{j+1}\right), \quad d\left(t_{j}\right), \quad d\left(\frac{t_{j}+t_{j+1}}{2}\right), \\
d\left(t_{j+1}\right), \quad j=1, \ldots, p-1 .
\end{gathered}
$$

We calculate the function

$$
R(t)=D\left(x_{a p p}(t)\right), t \in[a, b] .
$$

Next we solve the following problem : Find

$$
\begin{gathered}
x\left(t_{j}\right), \quad x\left(\frac{t_{j}+t_{j+1}}{2}\right), \quad x\left(t_{j+1}\right), \quad d\left(t_{j}\right), d\left(\frac{t_{j}+t_{j+1}}{2}\right), \\
d\left(t_{j+1}\right), \quad j=1, \ldots, p-1,
\end{gathered}
$$

minimizing

$$
J=\int_{a}^{b} R^{2}(t) d t, a \leq t \leq b
$$

while taking into account the restrictions :

$$
g_{i}\left(x^{(1)}(a), x^{(1)}(b), x(a), x(b), a, b\right)=0, i=1,2 .
$$

We replace

$$
x\left(t_{j}\right), x\left(\frac{t_{j}+t_{j+1}}{2}\right), x\left(t_{j+1}\right), d\left(t_{j}\right), d\left(\frac{t_{j}+t_{j+1}}{2}\right), \quad d\left(t_{j+1}\right),
$$

(obtained by minimization) in the expression of $x_{a p p}$ and we compute the corresponding value of $R$.

If $|R(t)|<\varepsilon$, then evidently $x_{a p p}$ is an $\varepsilon$ - approximate polynomial solution of the problem $(1,2)$.

\section{NUMERICAL EXAMPLE}

We consider the Liénard - Van der Pol equation together with the following boundary conditions :

$$
\left\{\begin{array}{c}
\ddot{x}+g_{1}(x) \dot{x}+g_{2}(x)=0 \\
x(0)=0 \\
\dot{x}(1)=1
\end{array}\right.
$$

on the interval $[0,1]$, where $g_{1}(x)=x^{2}-1, g_{2}(x)=x$. This equation has applications in Kaldor's Model in economy (Tu Pierre, 1992).

We consider the case of an approximation consisting of a single 5th degree polynomial. We obtain the 5th degree polynomial approximate analytical solution :

$$
\begin{aligned}
& x_{a p p}(t)=0.757242 t+0.343525 t^{2}+0.132281 t^{3} \\
& -0.265448 t^{4}+0.0324004 t^{5} .
\end{aligned}
$$

The following plot contains the graphical representation of this polynomial (solid line) together with the corresponding numerical solution of eq. (6) computed using the software Wolfram Mathematica 6 (dashed line).

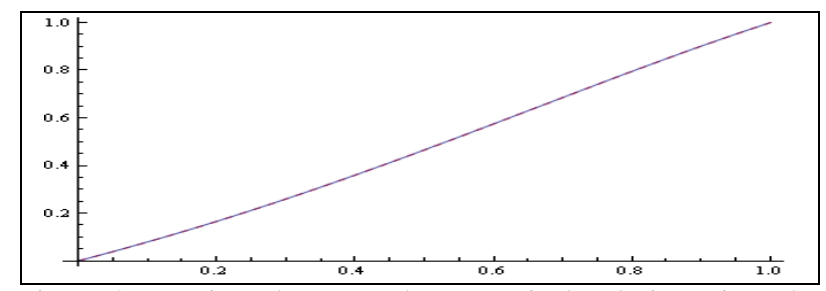

Fig.1. Comparison between the numerical solution given by Mathematica (dashed line) and the 5th degree polynomial approximation (solid line)

The difference between the two solutions (numerical and 5th degree polynomial approximate analytical) is smaller than 0.000121217 .

The graphical representation of the error $R$ is :

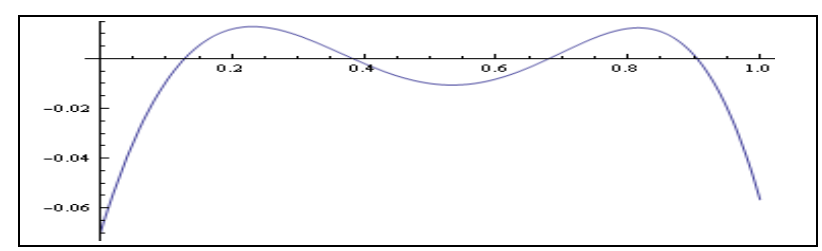

Fig. 2. Graphical representation of the error for the case of a 5th degree polynomial approximation

\section{CONCLUSIONS}

In this paper we presented a new method for the calculation of an analytical polynomial approximate solution for nonlinear problems of the type $(1,2)$.

For the numerical example presented here we used 5th degree polynomials, but if a higher precision is needed, polynomials of higher degree can be easily computed.

The main limitation of the method consists in the fact that it is taylored for the specific form of equations of the type $(1,2)$, which leads to a loss of generality.

In this regard, a future research direction could be the adaptation of the method for nonlinear differential equations of degree greater than two. Also, since a modified version of the method seems to be applicable to certain partial differential equations, another research direction is the development of a similar method for the case of partial differential equations.

\section{REFERENCES}

Chen, C.J.; Sheikholeslami, M.Z. \& Bhiladhvala, R.B. (1989). Finite analytic numerical method for two-point boundary value problems of ordinary differential equations, Computational Methods in Applied Mechanics and Engineering. Vol. 75, p. 61-76, ISSN: 00457825

Chena, B.; Garcia-Bolos, R.; Jodal, L. \& Rosello, M.D. (2005). Chebyshev polynomial approximations for nonlinear differential initial value problems, Nonlinear Analysis TMA, vol. 64, p.629-673, ISSN: 0362546X

Gotovac, H.; Kozulic, V.; Gotovac, B.; Sesartic, R.; Brajcic, N. \& Colak, I. (2009). Adaptive Fup Collocation Method for Time Dependent Partial Differential Equations, Annals of DAAAM for 2009 \& Proceedings of the 20th International DAAAM Symposium, 25-28th November 2009, Vienna, Austria, ISSN 1726-9679, ISBN 978-3-901509-70-4, Katalinic, B. (Ed.), pp. 1889-1890, Published by DAAAM International Vienna, Vienna

He, J.-H. (2003). Homotopy perturbation method : a new nonlinear analytical technique, Applied Mathematics and Computation, vol. 135, p.73-79, ISSN: 00963993

Sahmsul Alam, M.; Abul Kalam, M. \& Hoque, M.A. (2006). A general Struble's technique for solving a n-th order weakly non-linear differential system with damping, International Journal of Nonlinear Mechanics, vol. 41 , p. 905-918, ISSN: 00207462

Tu Pierre, N.V. (1992). Dynamical Systems - An introduction with applications in economics and biology, SpringerVerlag, Berlin, ISBN $10: 3540557806$ 